\title{
Taste interaction between glucose and fructose assessed by functional measurement
}

\author{
CEES DE GRAAF, JAN E. R. FRIJTERS, and HANS C. M. VAN TRIJP \\ Agricultural University, Wageningen, The Netherlands
}

\begin{abstract}
The description of the sensory interaction between two taste substances in terms of numerical responses obtained under a magnitude estimation instruction is biased, because the sensory processes are confounded with the judgmental process. Because the judgmental process is irrelevant to the sensory processes involved in the perception of taste substance mixtures, a correct description of mixture interaction can be obtained only with an experimental setup that separates the sensory processes from the judgmental process. Functional measurement in combination with a two-stimulus procedure can separate these two mechanisms. When this approach is used, parallelism in the factorial plot of the responses depends not on the underlying sensory processes, but on the comparative operation between two sensory impressions and on the form of the judgment function. In this experiment, solutions of glucose, three equiratio mixture types of glucose and fructose (i.e., mixtures in which the ratio of the components is constant), and fructose were compared with glucose solutions for sweetness intensity. Under the assumption that the comparative operation between two perceived sweetness intensities is subtractive, this scaling procedure yields interval scales of perceived sweetness intensity. The results showed that the data obtained are reliable, and that the psychophysical functions for equiratio mixtures of glucose and fructose lie in between the psychophysical functions for unmixed glucose and fructose.
\end{abstract}

Sensory interaction in mixtures of two qualitatively similar taste substances has frequently been studied using the method of magnitude estimation (e.g., Curtis, Stevens, \& Lawless, 1984; Frijters, De Graaf, \& Koolen, 1984; Frijters \& Oude Ophuis, 1983; Moskowitz, 1971, 1973, 1974a, 1974b; Rifkin \& Bartoshuk, 1980; Stone \& Oliver, 1969; Stone, Oliver, \& Kloehn, 1969). The use of this procedure, in conjunction with describing sensory interactions in terms of suppression, addition, and synergism, may lead to erroneous conclusions about the nature and magnitude of the taste interaction due to scaling artifacts. This is a major contention of this paper, and is substantiated below.

Magnitude estimation, as developed by Stevens (1956), originates from the stimulus-response (S-R) conception of psychophysics; it is assumed that the overt response is a perfect external representation of the internal sensation (McKenna, 1985; Shepard, 1981). In contrast, the stimulus-organism-response (S-O-R) paradigm of psychophysical judgment identifies a psychophysical stage relating stimulus to sensation and a judgmental stage relating sensation to overt response (Attneave, 1962; Torgerson, 1961; Treisman, 1964). Investigators who have adopted the S-O-R view have shown that the judgment function in magnitude estimation is a nonlinear and positively accelerating function of the internal sensation (e.g., Curtis, Attneave, \& Harrington, 1968; Rule \& Curtis, 1977; Rule, Curtis, \& Markley, 1970; Veit, 1978; Weiss, 1972).

The authors are indebted to Norman H. Anderson for his comments on this paper. Correspondence should be addressed to C. de Graaf, Department of Human Nutrition, De Dreijen 12, 6703 BC Wageningen, The Netherlands.
This implies that the description of the sensory interaction between two taste substances, in terms of numerical responses obtained by magnitude estimation, is biased by the nonlinear judgment function. This point can be elucidated by the following example. Suppose that concentration $x \mathrm{M}$ of substance $\mathrm{A}$ has a taste intensity of 5 units on a ratio scale, and that concentration $y \mathbf{M}$ of substance $B$ has a taste intensity of 10 units, also on a ratio scale. If the two taste substances behave additively when mixed, then the taste intensity of a mixture containing $x \mathbf{M}$ of $\mathrm{A}$ plus $y \mathrm{M}$ of $\mathrm{B}$ should have a taste intensity of 15 units. If these three stimuli ( $x \mathrm{M}$ of $\mathrm{A}, y \mathrm{M}$ of B, and $x \mathrm{M}$ of $\mathrm{A}+y \mathrm{M}$ of $\mathrm{B}$ ) were presented in a magnitude estimation experiment in which the response function has the form (Perceived Intensity) $)^{1.47}(1.47$ being the mean value for the exponent of the response function found in magnitude estimation experiments; Birnbaum, 1980), then the reported magnitude of $x \mathrm{M}$ of $\mathrm{A}$ would be 11 , that of $y \mathrm{M}$ of $B$ would be 30 , and the response to the mixture would be 54. From this result it would then erroneously be concluded that substances A and B behave synergistically when mixed, because the number 54 is larger than the sum of 30 and 11 . This example demonstrates that in magnitude estimation, the sensory processes involved in the perception of the taste intensity of a mixture are confounded with the judgmental process. Since the judgment operation is irrelevant to the sensory interaction, a correct description of the sensory processes involved in the perception of taste substance mixtures can be achieved only by a measurement procedure that separates the sensory processes from the judgment function. We believe that a functional measurement approach in combination 
with a two-stimulus procedure (Anderson, 1981, 1982) is appropriate to achieve this goal.

The aim of the present experiment was to investigate the sensory interaction between two qualitatively similar taste substances, with a measurement procedure yielding overt responses that are a linear function of the perceived taste intensities. Since the psychophysics of taste mixtures has rarely been studied with a functional measurement procedure (Klitzner, 1975; McBride, 1986), some additional explanation is given first.

\section{FUNCTIONAL MEASUREMENT AND MIXTURES OF TASTE SUBSTANCES}

One of the main features of functional measurement is the use of factorial designs as a tool for the evaluation of the form of the judgment function (Anderson, 1981). For the investigation of mixtures of taste substances, two different factorial designs can be constructed: one at a physical level and the other at a judgmental level. There is a fundamental difference between these two factorial designs.

A physical factorial design does not help to resolve the entanglement between sensory interaction and the judgment function. In this type of experimental design, parallelism in a factorial plot of responses is obtained if two conditions are satisfied. The first condition is that the two tastants in a mixture contribute in an additive way to the total taste intensity of the mixture. The second condition is that the judgment function is linear with perceived taste intensity. If, and only if, taste additivity holds, parallelism in a factorial plot signifies linearity of the judgment function. To put it differently, nonparallelism in a factorial plot can result from three different causes: (1) Tastants used for the mixture composition behave nonadditively; (2) responses are not linear with perceived taste intensity; or (3) a combination of 1 and 2 . The contention that a factorial design at a physical level does not help to separate sensory interaction from the cognitive judgmental operation is based on the logical impossibility of distinguishing among these three causes.
The use of a factorial design at a judgmental level prevents the confounding between sensory interaction and the judgmental operation. Such a procedure allows for the determination of the shape of the judgment function independently from the nature of the sensory interaction that occurred. This argument is substantiated below.

McBride $(1982,1986)$ applied functional measurement to mixtures of taste substances. In a series of five experiments he investigated whether sucrose, fructose, and glucose in binary mixtures contribute in an additive way to the perceived sweetness intensity of those mixtures. Figure 1 diagrams McBride's experimental setup. The factorial design in these experiments was constructed at the physical level; each of a number of concentrations of sucrose, fructose, and glucose was mixed with each of a number of concentrations of one of the other sugars, yielding sucrose-fructose, sucrose-glucose, and fructoseglucose mixtures. We call this a factorial mixing design. The perceived sweetness intensities of single stimuli, each comprising two substances, were rated on a 13-point category scale. McBride found that the factorial plots of the obtained data in the five experiments did not exhibit sets of parallel lines: All plots showed a convergent and significant interaction between the two sugars constituting the mixtures. However, due to the nature of this type of experimental design, it is logically impossible to separate nonparallelism resulting from a nonlinear judgment function from nonparallelism resulting from a nonadditive sensory integration. As Figure 1 shows, in this type of experimental design the judgment function is still confounded with the sensory processes involved when two tastants are mixed.

Disentanglement of the sensory processes and the judgmental process can be achieved by a modified experimental setup that makes use of a two-operation model (see Anderson, 1974, Table II). This approach was first used in taste psychophysics by Klitzner (1975), who investigated whether the preference structure for mixtures of apple juice and a bitter substance could be described by an additive integration model. Klitzner found that the lines in the factorial plots of the responses converged, which

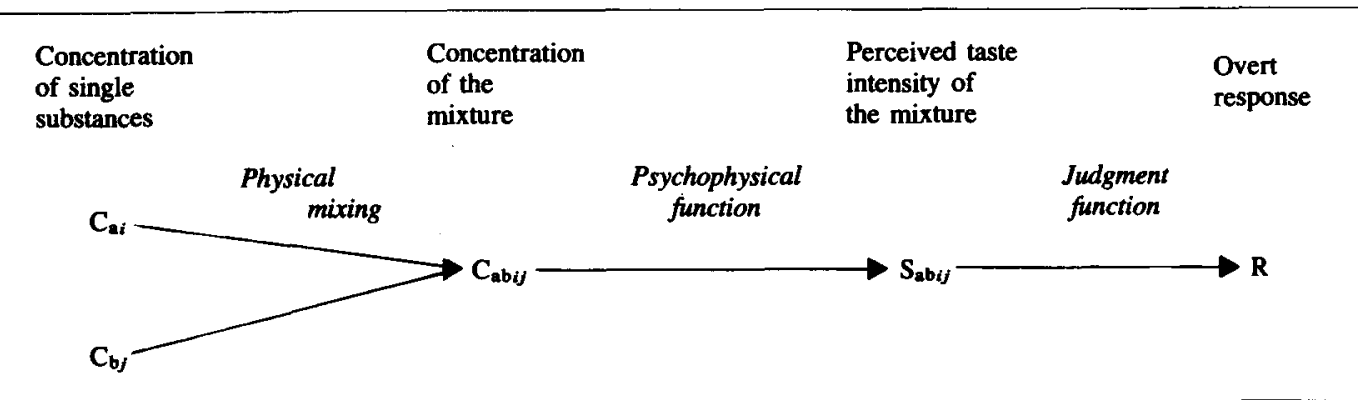

\footnotetext{
Figure 1. Outline of McBride's (1982, 1980) experimental setup from a stimulus-organism-response (S-O-R) point of view. Each concentration $i$ of substance $A$ is mixed with each concentration $j$ of substance $B$ (i.e., a factorial mixing design), resulting in concentration $C_{a b i j}$. The mixture concentration $C_{a b i j}$ evokes perceived taste intensity $S_{a b i j}$. The judgment function transforms $S_{a b y}$ into the overt response $R$.
} 
means that there was a significant interaction. However, as argued above, he was not in a position to decide whether this interaction resulted from the taste interaction or from a taste-hedonic interaction between the apple juice and the bitter substance. A third possibility would be a nonlinear relationship between preference and response. To determine the origin of the observed nonparallelism, he subsequently introduced a second judgmental step in the psychological model. In a second experiment, Klitzner asked subjects to judge the difference in preference between two bitter-substance/apple-juice mixtures. The parallelism of the lines in the factorial plot of the responses in this experiment demonstrated that the preference-response function was linear. On the basis of this result, Klitzner argued that the interaction in the factorial plot in the first experiment had most probably been due to a nonadditive taste interaction or to a taste-hedonic interaction between apple juice and the bitter substance.

Two-operation models in psychophysics have been extensively investigated by Birnbaum and colleagues (Birnbaum \& Elmasian, 1977; Birnbaum \& Mellers, 1978; Birnbaum \& Veit, 1974; Hagerty \& Birnbaum, 1978; Mellers, Davis, \& Birnbaum, 1984; Veit, 1978). In these experiments, a factorial design was not used at a physical level, as it was in McBride's $(1982,1986)$ experiments, but it was used at a judgmental level (i.e., a factorial judgment design). The subjects compared the sensory impression of each level of the row stimulus with the sensory impression of each level of the column stimulus. Figure 2 illustrates this type of experimental setup. A major advantage of this setup is that parallelism or nonparallelism in the factorial plot of the data obtained does not depend on the underlying sensory processes (including the psychophysical functions). Parallelism or nonparallelism in this type of experiment depends on the na- ture of the comparative operation between two sensory impressions and also on the form of the judgment function; it cannot depend on sensory interaction, because the stimuli used are not compound stimuli. From four studies carried out with such a two-stimulus procedure, it was concluded that the comparative operation between two sensory impressions can be best described by an algebraic subtractive rule, even when subjects are instructed to judge ratios (Birnbaum \& Mellers, 1978; Hagerty \& Birnbaum, 1978; Mellers et al., 1984; Veit, 1978).

Application of this approach to taste mixtures implies that the perceived taste intensity of each of a number of concentrations of the row stimulus should be compared with the perceived taste intensity of each of a number of concentrations of the column stimulus. Because we are dealing with taste mixtures, the row and column stimuli may consist of mixtures of taste substances, instead of unmixed tastants. The question then arises of how to deal with the physical composition of these mixtures. In most experiments, the physical intensity of a stimulus is varied in one dimension (e.g., weight in $\mathrm{kg}$, sound pressure in $\mathrm{N} / \mathrm{m}^{2}$ ), whereas in mixtures of taste substances there are two stimulus dimensions that are being manipulated (i.e., the concentrations of each of two substances in a binary mixture). This problem can be handled by conceiving of a binary mixture of taste stimuli as if it were one particular type of taste stimulus; this was done by Frijters and Oude Ophuis (1983), who introduced the concept of the equiratio taste substance mixture. An equiratio mixture type was defined as a series of taste substance mixtures in each of which the ratio of the components is constant. Frijters and Oude Ophuis showed that a psychophysical function of an equiratio mixture type can be experimentally determined in the same way as such a function for a single substance. The measure of physical intensity of

\begin{tabular}{llll}
$\begin{array}{l}\text { Concentration } \\
\text { of single } \\
\text { substances }\end{array}$ & $\begin{array}{l}\text { Concentration } \\
\text { of the } \\
\text { mixtures }\end{array}$ & $\begin{array}{l}\text { Perceived taste } \\
\text { intensity of } \\
\text { the mixtures }\end{array}$ & $\begin{array}{l}\text { Integrated } \\
\text { impression }\end{array}$ \\
\hline
\end{tabular}

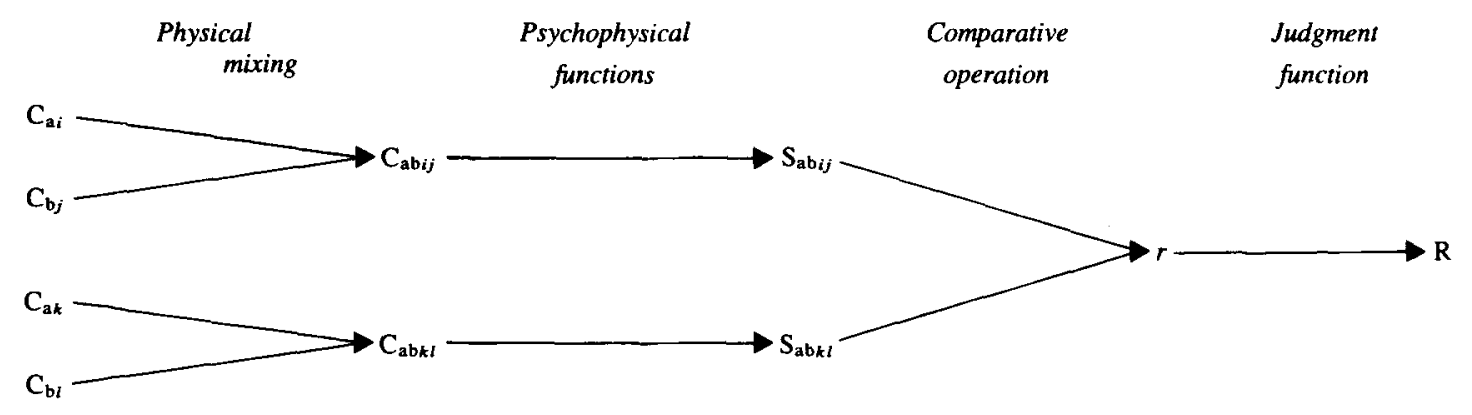

Figure 2. Outline of the present experimental setup from a stimulus-organism-response (S-O-R) point of view. Concentration $i$ of substance $A$ is mixed with concentration $j$ of substance $B$, resulting in mixture concentration $C_{a b i j}$. Mixing of $k$ of $A$ and $l$ of $B$ results in mixture concentration $\mathrm{C}_{\mathrm{ab} k l}$. Both equiratio mixtures (i.e., $i / j=$ constant and $k / l=$ constant) and single substances (i.e., either $i$ or $j=0$ or $k$ or $l=0$ ) were used. The concentrations $C_{a b i j}$ and $C_{a b k l}$ evoke perceived taste intensities $S_{a b i j}$ and $S_{a b k l}$, respectively. If the comparative operation between two perceived taste intensities can be represented by an algebraic difference function, then the integrated impression $r$ resembles the perceived difference between $S_{a b i j}$ and $S_{a b k i}$. Each $S_{a b_{i j}}$ is compared with each $S_{a b k l}$ (i.e., a factorial judgment design). The judgment function transforms $r$ into the overt response $R$. 
a mixture is the total concentration of the mixture (i.e., the sum of the concentrations of the mixture's components). Figure 2 diagrams the present approach to the psychophysics of taste mixtures. A complete factorial design in such an experiment consists of a comparison of the perceived taste intensity of each level of a mixture having concentration $i$ of substance $\mathrm{A}$ and concentration $j$ of substance $B\left(C_{a b i j}\right)$ with the perceived taste intensity of each level having concentration $k$ of substance $A$ and concentration $l$ of substance B $\left(\mathrm{C}_{\mathrm{abk} l}\right)$.

To draw a logically sound conclusion about the sensory interaction between two taste substances, we must compare the perceived taste intensities of the mixture with the perceived taste intensities of the mixture's unmixed components. For that reason, concentrations of the single substances (i.e., either $i$ or $j=0$, or $k$ or $l=0$ ) also have to be incorporated in the factorial judgment design. The experimental setup in the present study consisted of a series of factorial designs, in each of which the perceived taste intensity of each of a number of mixtures was compared with the perceived taste intensity of each of a number of concentrations of an unmixed substance.

If it is assumed that the comparative operation between two perceived taste intensities follows a subtractive rule, then parallelism in the factorial plot of the obtained responses implies that the judgment function is linear with sweetness differences. If this is the case, it is possible to obtain scale values for the perceived intensities of mixtures of taste substances (or single substances) on linear scales, and psychophysical functions can be constructed subsequently.

\section{METHOD}

\section{Subjects}

Twelve paid volunteers were used, 10 women and 2 men, ranging in age from 18 to 25 years. All subjects were graduate or undergraduate students from the Agricultural University. Most of the subjects had had previous experience with psychophysical tasks, but all were naive with respect to the substances used and the purpose of the study.

\section{Stimuli}

The stimuli were solutions of glucose (Merck: 15639), fructose (Merck: 5321), and three equiratio mixture types in demineralized water. The equiratio mixture types comprised mixtures containing three times as much glucose as fructose (GluFru 0.75/0.25), mixtures with an equal concentration of both substances (GluFru $0.50 / 0.50$ ), and mixtures containing three times as much fructose as glucose (GluFru 0.25/0.75). Solutions were prepared at least $24 \mathrm{~h}$ before tasting and stored for no longer than 5 days at $4^{\circ} \mathrm{C}$. The concentrations used were the same for each of the five stimulus types: 0.000 (water), $0.125,0.25,0.50,1.00$, and $2.00 \mathrm{M}$. Water and $2.50-\mathrm{M}$ fructose served as the reference pair.

\section{Design}

Glucose, GluFru 0.75/0.25, GluFru 0.50/0.50, GluFru 0.25/0.75, and fructose were all compared with glucose in nine separate 6 (concentrations of the first stimulus) $\times 6$ (concentrations of the second stimulus) factorial designs. In four factorial designs glucose served as the first stimulus and either fructose or one of the three equiratio mixture types was the second stimulus, in four other designs the converse sequence was used, and in one design both the first and second stimulus contained glucose only.

\section{Procedure}

The subjects were instructed to judge the magnitude of the difference in perceived sweetness intensity between the first and second stimulus within each pair. The instructions emphasized that only the sweetness intensity was to be judged, and that both the hedonic value and side tastes were to be disregarded. The judgments were to be expressed by a slash mark on a $250-\mathrm{mm}$ symmetric scale (see Figure 3), of which the middle (having the assigned value of 125) was defined as "the first and second stimulus are equal in sweetness intensity" (no difference). When the second stimulus was perceived as sweeter than the first stimulus, the subjects were to mark a slash on the right-hand side of the scale. When the first stimulus was perceived as sweeter than the second, the subjects were to mark a slash on the left-hand side of the scale. In the instructions, the term maximum difference was defined as the difference between the perceived sweetness intensities of the stimuli within the reference pair (water as the first stimulus and a 2.5-M fructose solution as the second stimulus). The difference in this pair was expected to be larger than the difference in any other pair to be judged in the experiment.

The subjects were requested to rinse their mouths thoroughly with demineralized water both between the two stimuli within a pair and between pairs. The stimuli were presented at room temperature in polystyrene medicine cups, each cup containing about $10 \mathrm{ml}$ of solution. Each factorial design was composed of $36(6 \times 6)$ pairs, presented in a random sequence and in a different order for each subject. The reference pair was presented at the beginning of each session and again after the 12th and 24th pair of a series. In a pilot study, it had been determined that a time interval of $50 \mathrm{sec}$ between the first and second stimulus of a pair was sufficient to prevent adaptation. The time interval between pairs was also $50 \mathrm{sec}$. Each of the nine series of 36 pairs was tasted three times by each subject.

Difference in sweetness intensity between first and second stimulus

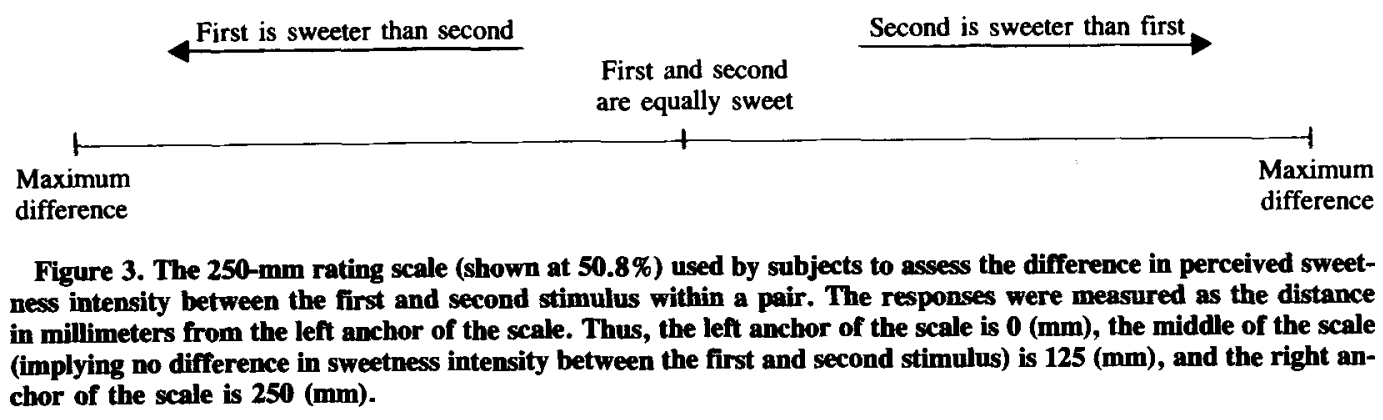


The order of presentation of the various series was randomized. In each session, one series of 36 pairs was judged by each subject, so it took each subject 27 sessions to complete the experiment.

\section{RESULTS}

To draw conclusions about the sensory interaction between glucose and fructose, it is necessary to derive one scale value for each stimulus tasted. This scale value should represent the perceived sweetness intensity on a linear scale. However, before these scale values can be calculated, it must be determined whether or not the judgment function is linear.

\section{The Judgment Function}

The subjects were instructed to judge the difference between the perceived taste intensities within each pair of stimuli. If it is assumed that subjects have indeed judged a difference (i.e., $r=S_{a b i j}-S_{a b k l}$; see Figure 2), parallelism in a factorial plot implies that the response function is linear with the perceived difference (i.e., $\mathrm{R}=\mathrm{a}+\mathrm{b} r$ ). The term factorial plot refers to a plot of reported differences (not of the perceived taste intensities themselves) against the concentration of the second stimulus with a separate curve for each concentration of the first stimulus.

Because individuals may vary in their (linear or nonlinear) judgment functions, or in their comparative operations (which may or may not be subtractive), we first performed individual analyses. To test whether parallelism was actually obtained, an analysis of variance was carried out for each subject and each type of design (i.e., $12 \times 9=108$ analyses). The measure of the degree of nonparallelism, that is, the row $\times$ column interaction, was tested for statistical significance, with the row $\times$ column $x$ replicate interaction as error term. Two subjects showed significant row $\times$ column interactions at the $p<.01$ level $[F(25,50) \geq 2.12]$ in two or more of their nine response matrices. Apparently, either these subjects had nonlinear response functions or their comparative operations were not subtractive. The data derived from these subjects were excluded from further data analyses. In the analyses of the 10 remaining subjects, no significant interactions at the $p<.01$ level were obtained.

After these individual analyses, the responses for each pair were averaged across the three replicates and the 10 subjects. Figure 4 shows the arithmetic mean of the responses to each pair for each of the nine factorial designs. Analyses of variance for each of the nine response matrices showed no significant row $\times$ column interactions (see upper left part of each panel in Figure 4). The error term for this interaction in these group analyses was the row $\times$ column $\times$ subject interaction. The six curves within each of the nine panels show no systematic deviations from parallelism; therefore, it can be concluded that the responses are linear with sweetness differences.

It should be noted that, in principle, parallelism in a factorial plot can also be obtained by a nonsubtractive comparative operation in combination with a nonlinear judgment function, for example, a ratio operation in combination with a logarithmic judgment function (Birnbaum, 1982). It is logically impossible to decide from the data which combination of comparative operation and judgment function is used; this is the problem of "monotonic indeterminacy" (Anderson, 1974). However, considering that the subjects were explicitly instructed to make difference judgments, and in view of a substantial body of empirical evidence supporting a difference operation (Birnbaum \& Mellers, 1978; Hagerty \& Birnbaum, 1978; Mellers et al., 1984; Veit, 1978), it is likely that the observed parallelism in the present experiment resulted from the use of difference judgments in combination with linear judgment functions.

\section{Scale Values of Perceived Sweetness Intensity}

The observed parallelism also implies that the marginal means of the row (first) and column (second) stimuli are valid estimates of the sweetness intensities of the corresponding row and column concentrations on linear scales (Anderson, 1981). As water was incorporated in each of the series of row and column stimuli, the scale value of water can be used as a conventional point of reference. The perceived sweetness intensity of each stimulus can then be defined as the distance between the scale value of that stimulus and the scale value of water. For practical purposes, the scale value of water was set equal to zero. If it is assumed that water lacks a sweet taste, then this point might be treated as an absolute zero point for the scale of perceived sweetness intensity, implying that the derived scale is a ratio scale. However, it appears that additional evidence is required before we can claim that the developed scale does indeed have ratio scale properties.

Table 1 shows, for illustrative purposes, the derivation of scale values for sweetness intensity for the fructoseglucose factorial judgment design. Each factorial design yielded two marginal means for water-one for water as the first stimulus in a pair, and one for water as the second stimulus. The scale values for each of the sugarcontaining row stimuli were determined by calculating the difference between their respective marginal means and the marginal mean of water in that same design. The scale values of the column stimuli were determined in a similar way. These calculations yielded 10 sets of scale values for glucose -5 sets for glucose as the first stimulus (of each pair) and 5 sets for glucose as the second stimulus (of each pair). For fructose and each of the three equiratio mixture types, two sets of scale values were derived-one set for each stimulus when presented as the first stimulus, and one set for each stimulus when presented as the second stimulus. The scale values for each row (first) stimulus were virtually identical to the scale values for the same stimulus when it was used as column (second) stimulus. This result indicates an absence of order effects.

The conclusion that there were no order effects can also be reached through another line of reasoning. Each stimu- 


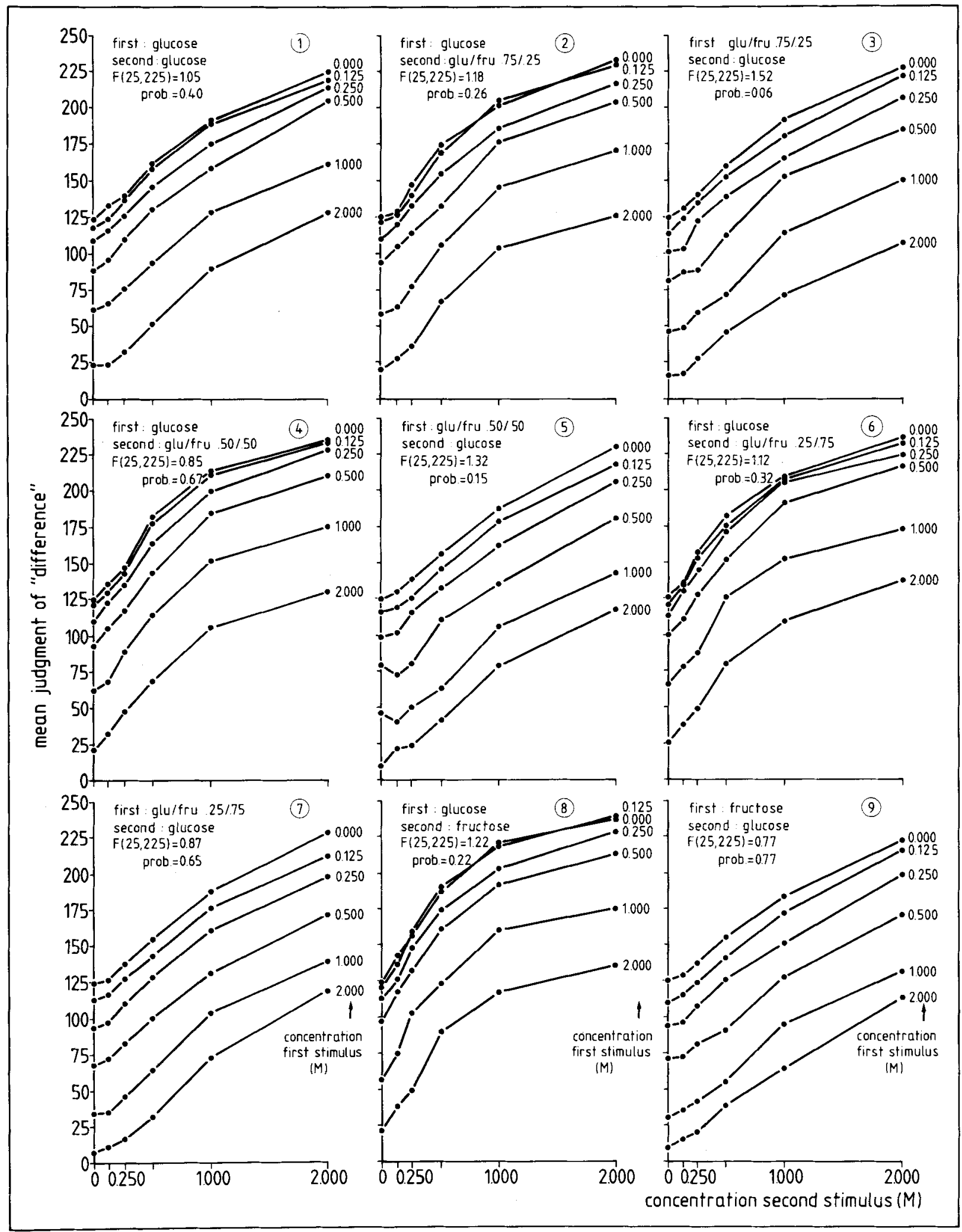

Figure 4. Mean reported judgment of difference in perceived sweetness intensity between the first and second stimulus within a pair. Each panel represents a different factorial judgment design. The substances used and the $F$ ratio for the row (first) $\times$ column (second) interaction are given in the upper left part of each panel. 
Table 1

Derivation of Ratio Scale Values of Perceived Sweetness Intensity

\begin{tabular}{|c|c|c|c|c|c|c|c|c|}
\hline \multirow{2}{*}{$\begin{array}{c}\text { Concentration (M) } \\
\text { of the First } \\
\text { Stimulus (Fructose) }\end{array}$} & \multicolumn{3}{|c|}{ Concentration (M) of the } & \multicolumn{3}{|c|}{ Second Stimulus (Glucose) } & \multirow{2}{*}{$\begin{array}{c}\text { Marginal } \\
\text { Mean First } \\
\text { Stimulus }\end{array}$} & \multirow{2}{*}{$\begin{array}{c}\text { Distance to } \\
\text { Scale Value } \\
\text { of Water }\end{array}$} \\
\hline & 0.000 & 0.125 & 0.250 & 0.500 & 1.000 & 2.000 & & \\
\hline 0.000 & 125.37 & 129.27 & 137.93 & 154.70 & 183.60 & 223.33 & 159.03 & 0.00 \\
\hline 0.125 & 110.10 & 115.33 & 122.90 & 140.73 & 173.37 & 215.90 & 146.39 & 12.64 \\
\hline 0.250 & 94.53 & 96.13 & 107.57 & 124.60 & 151.03 & 199.17 & 128.84 & 30.19 \\
\hline 0.500 & 71.30 & 71.63 & 75.93 & 90.53 & 128.33 & 171.87 & 101.60 & 57.30 \\
\hline 1.000 & 31.87 & 36.63 & 41.53 & 55.20 & 95.47 & 131.97 & 65.44 & 93.59 \\
\hline 2.000 & 9.87 & 16.07 & 20.07 & 38.03 & 64.07 & 113.33 & 43.75 & 115.46 \\
\hline $\begin{array}{l}\text { Marginal mean } \\
\text { second stimulus }\end{array}$ & 73.84 & 77.51 & 84.32 & 100.63 & 132.64 & 175.93 & & \\
\hline $\begin{array}{l}\text { Distance to scale } \\
\text { value of water }\end{array}$ & 0.00 & 3.67 & 10.49 & 26.79 & 58.80 & 102.09 & & \\
\hline
\end{tabular}

Note-The figures in the response matrix represent the mean reported difference between the first (row) stimulus and second (column) stimulus within each pair of the fructose(first)-glucose(second) factorial judgment design.

lus was presented the same number of times as the first and as the second stimulus. Without an order effect, the overall arithmetic mean of the responses should be 125 (i.e., the middle of the scale). If the first stimulus had a greater weight than the second stimulus in determining the response, the overall mean would be lower than 125 . Similarly, if the second stimulus had a greater weight, the overall mean would be higher than 125 . The means (averaged over $9 \times 36 \times 3=972$ responses) for each of the 10 subjects were $126.0,123.1,124.0,127.0,125.4$, $125.2,126.8,126.5,126.7$, and 125.6. The value of the standard errors of the means is about 2 , so none of these values deviates significantly from 125 . Therefore, it can be safely concluded that no order effects occurred.

The final scale value for each stimulus was obtained by calculating the arithmetic mean of the scale value(s) for that stimulus when tasted first and the scale value(s) of that stimulus when tasted second. The final scale values of each stimulus are shown in Figure 5.

Bogartz (1980) proposed an analysis of variance procedure to test whether the row psychophysical function is the same as the column psychophysical function. How-

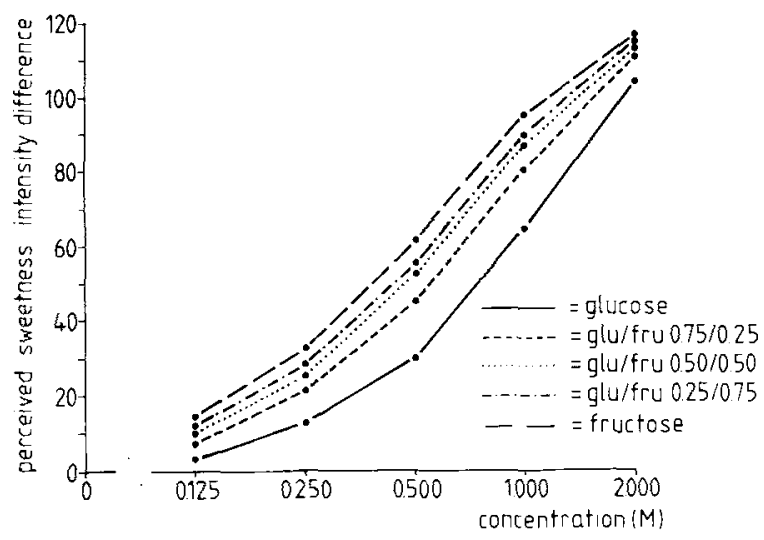

Figure 5. Scale values of the differences in perceived sweetness intensity between water and glucose, fructose, and the three equiratio mixture types. ever, this test can be applied only to designs in which the series of row stimuli and the series of column stimuli are the same. In the present study, this was the case for the glucose-glucose design only. Using a second-order polynomial function as an approximation of the unknown psychophysical function (Bogartz, 1980), we performed an analysis of variance on the means of the overt responses for the glucose-glucose design. No difference was found between the psychophysical function for glucose tasted first (row) and the psychophysical function for glucose tasted second (column) $[F(2,31)=0.81, p>.5]$. This provides additional justification for averaging the scale values of the row and column stimuli.

\section{Comparison With Matching Data}

The reliability (not the validity) of the present data was verified through comparison of the present data with data on glucose-fructose mixtures obtained by matching in an earlier experiment (De Graaf \& Frijters, 1986). In that experiment, fructose, GluFru $0.75 / 0.25$, GluFru $0.50 / 0.50$, and GluFru $0.25 / 0.75$ were matched in perceived sweetness intensity to $0.125-, 0.25-, 0.50-, 1.00$-, and 2.00-M glucose, using the method of constant stimuli. These data were shown to be accurate: the points of subjective equality (PSEs) in 10 control experiments had a mean absolute error of $0.64 \%$.

The PSEs in the present experiment were calculated as follows. For fructose, GluFru 0.25/0.75, GluFru $0.50 / 0.50$, and GluFru $0.75 / 0.25$, second-order polynomial regression equations were fitted with $\log$ (concentration) and $[\log (\text { concentration })]^{2}$ as independent variables and the $\log$ of the final scale values as a dependent variable. All fitted functions had an $R^{2}$ of 0.9998 or higher, and were monotone with the relevant domain. The obtained equations were set equal to the log of the five scale values of glucose concentrations, and the resulting quadratic equations were resolved for the $\log$ of the required concentration.

Table 2 shows that the PSEs determined from the present data have an average absolute deviation of about 
Table 2

Comparison of Points of Subjective Equality (PSEs) Determined Using the Method of Constant Stimuli* and PSEs Calculated From the Data of the Present Study

\begin{tabular}{clccc}
\hline $\begin{array}{c}\text { Concentration of } \\
\text { Glucose (M) }\end{array}$ & $\begin{array}{c}\text { Type of } \\
\text { Comparison Stimulus }\end{array}$ & $\begin{array}{c}\text { PSE Determined } \\
\text { From Matching (M) }\end{array}$ & $\begin{array}{c}\text { PSE Determined From } \\
\text { Present Data (M) }\end{array}$ & $\begin{array}{c}\text { Difference in } \%_{\text {PSE }} \text { matching } \\
\text { PSE }\end{array}$ \\
\hline 0.125 & Fructose & 0.0485 & 0.0480 & -1.0 \\
0.125 & GluFru 0.25/0.75 & 0.0540 & 0.0558 & +3.3 \\
0.125 & GluFru 0.50/0.50 & 0.0650 & 0.0666 & +2.5 \\
0.125 & GluFru 0.75/0.25 & 0.0873 & 0.0772 & -11.6 \\
0.250 & Fructose & 0.1027 & 0.1038 & +1.0 \\
0.250 & GluFru 0.25/0.75 & 0.1206 & 0.1298 & +7.6 \\
0.250 & GluFru 0.50/0.50 & 0.1439 & 0.1486 & +3.2 \\
0.250 & GluFru 0.75/0.25 & 0.1817 & 0.1739 & -4.3 \\
0.500 & Fructose & 0.2374 & 0.2275 & -4.0 \\
0.500 & GluFru 0.25/0.75 & 0.2682 & 0.2594 & -3.3 \\
0.500 & GluFru 0.50/0.50 & 0.3118 & 0.2862 & -10.8 \\
0.500 & GluFru 0.75/0.25 & 0.3639 & 0.3308 & -9.1 \\
1.000 & Fructose & 0.5790 & 0.5266 & -9.1 \\
1.000 & GluFru 0.25/0.75 & 0.5928 & 0.6013 & +1.4 \\
1.000 & GluFru 0.50/0.50 & 0.6550 & 0.6350 & -3.1 \\
1.000 & GluFru 0.75/0.25 & 0.7729 & 0.7221 & -6.5 \\
2.000 & Fructose & 1.3828 & 1.3038 & -5.7 \\
2.000 & GluFru 0.25/0.75 & 1.4007 & 1.4587 & +4.1 \\
2.000 & GluFru 0.50/0.50 & 1.4552 & 1.4841 & +1.9 \\
2.000 & GluFru 0.75/0.25 & 1.6310 & 1.6506 & +1.2 \\
& & & Mean absolute deviation: & 4.7 \\
\hline
\end{tabular}

*From De Graaf and Frijters (1986).

5\% from the PSEs determined from the matching data. There appears to be no systematic deviation; nearly half of the PSEs estimated from the present study are higher than those obtained from matching, and half are lower. The greatest deviation is $-11.6 \%$, which is less than one JND.

It should be borne in mind that the present psychophysical functions were established on the basis of the assumption that the scale value of water can be used as a conventional point of reference that can be set equal to zero. If this assumption were incorrect (e.g., if water evoked a different sensory impression when compared to glucose than when compared to fructose), systematic deviations between the two sets of PSEs would have resulted. Because there are no such deviations, the data of Table 2 support the validity of the assumption that water represents a meaningful zeropoint.

\section{Sensory Interaction Between Glucose and Fructose}

As Figure 5 shows, the scale values of equiratio mixtures lie in between the scale values of equimolar concentrations of unmixed glucose and unmixed fructose. Thus, the sweetness intensity of a particular mixture of glucose and fructose does not exceed the sweetness intensity of an equimolar concentration of fructose, nor is it less than the sweetness intensity of an equimolar concentration of glucose. When the proportion of fructose in a mixture increases, the scale value of the mixture approaches the scale value of an equimolar concentration of fructose. Similarly, when the proportion of glucose in a mixture increases, the scale value of the mixture approaches the scale value of an equimolar concentration of glucose.

\section{DISCUSSION}

The similarity of the present data, obtained by direct scaling, to the data obtained by matching (De Graaf \& Frijters, 1986) shows that the scaling approach proposed in this study results in reliable estimates of perceived sweetness intensity. In addition, the present methodology provides a means of separating the sensory processes involved when mixing two tastants from the judgmental process. It makes possible an unbiased description of the sensory interaction between glucose and fructose.

\section{The Sensory Interaction Between Glucose and Fructose}

One conclusion to be drawn from the present results is that the psychophysical functions of equiratio mixtures of glucose and fructose lie in between the psychophysical functions of unmixed glucose and fructose. When the proportion of fructose in a fructose-glucose mixture increases, the behavior of the mixture approaches the behavior of fructose. Similarly, when the proportion of glucose in such a mixture increases, the behavior of the mixture becomes more like that of glucose. Thus, the values of the parameters of the psychophysical functions of equiratio mixtures of glucose and fructose (irrespec- 
tive of their forms) lie in between the values of the parameters of the psychophysical functions of unmixed glucose and fructose. This conclusion confirms the results of Frijters and Oude Ophuis (1983), who used the same stimuli but used magnitude estimation as the scaling method.

The nature of the taste interaction between two taste substances is usually characterized in terms of suppression, addition, and synergism, meaning, respectively, that the perceived taste intensity of a mixture is less than, equal to, or greater than the sum of the intensities of the unmixed components (e.g., Stone \& Oliver, 1969; van der Heyden, Brussel, Heidema, Kosmeyer, \& Peer, 1983; Yamaguchi, Yoshikawa, Ikeda, \& Ninomiya, 1970). As Frijters (in press) noted, a description of the taste interaction in these terms requires that the perceived taste intensities be assessed on a ratio scale. Some investigators hold the view that the type of sensory interaction is an attribute of the components used for mixture composition. We do not share this view. Following Bartoshuk (1975, 1977), we take the position that the type of sensory interaction that occurs is dependent on the shape of the psychophysical functions of the mixture's constituents. However, knowing the magnitude of the exponent of these functions, which is often thought to give an adequate description of the form of the psychophysical function, is insufficient for predicting the type of taste interaction that will occur. Assuming that the present scale values resemble perceived sweetness intensities on a ratio scale, it would be concluded that at the low sweetness levels used in this experiment, glucose and fructose show synergism when mixed. A $0.125-\mathrm{M}$ glucose solution has a sweetness intensity of about 3 units (see Figure 5), a $0.125-\mathrm{M}$ fructose solution has a perceived sweetness intensity of 14 units, and a mixture of both concentrations (i.e., $0.25 \mathrm{M}$ of the GluFru 0.50/0.50 mixture) has a sweetness intensity of 25 units $(25>3+14)$. At intermediate levels, it would be concluded that addition takes place. A $0.50-\mathrm{M}$ glucose solution is 30 units sweet, a $0.50-\mathrm{M}$ fructose solution is 61 units sweet, and a mixture of these concentrations (i.e., $1.00 \mathrm{M}$ of the GluFru $0.50 / 0.50 \mathrm{mix}$ ture) is 88 units sweet, which is almost equal to the sum of the intensities of the unmixed components $(30+61=91)$. At high intensities, it would be concluded that glucose and fructose suppress each other, because the sweetness intensity of a mixture of $1.00-\mathrm{M}$ glucose and $1.00-\mathrm{M}$ fructose (i.e., $2.00 \mathrm{M}$ of the GluFru $0.50 / 0.50$ mixture) is 114 units, which is less than the sum of the intensities of the unmixed components (1.00-M glucose is 64 units sweet, and 1.00-M fructose is 90 units sweet). The kind of taste interaction is evidently related to the forms and local steepnesses of the psychophysical functions of glucose and fructose. These are positively accelerating at low concentrations and negatively accelerating at high concentrations.

The conclusion that glucose and fructose show synergism at low sweetness levels is not in line with the results of McBride (1986), who concluded that glucose and fructose show additivity at low sweetness levels. However.
McBride did not present unmixed glucose and fructose; he presented only mixtures. Therefore, in his study, the perceived taste intensity of a mixture could not be compared with the sum of the perceived taste intensities of the mixture's components outside the mixture. McBride's results therefore suggest an additive increment in sweetness intensity when the concentration of one of the two substances in the mixture is increased, at low sweetness levels only.

\section{Sensory Interaction in General}

The sweetness intensity of glucose-fructose mixtures always lies in between the sweetness intensities of equimolar concentrations of the unmixed components. This has also been observed for sucrose-sorbitol mixtures (Frijters et al., 1984) and mixtures of L-ascorbic acid and itaconic acid (Frijters \& Stevens, 1986). The same rule appears to apply for a host of pairs of sugars (De Graaf \& Frijters, 1987). Interpretation of these findings suggests a general rule applicable to binary mixtures of substances with similar tastes: The taste intensity of any mixture of $A$ and $B$ having a particular molarity lies in between the taste intensities of the components $A$ and $B$, each having the same molarity as that of the mixture. This rule, suggested earlier by De Graaf and Frijters (1987), is in contrast to McBride's (1986) notion that the sweetness intensity of sucrose-fructose mixtures may exceed the highest intensity of the unmixed components. His claim is based on the use of weight/volume as the measure of concentration. When McBride's comparison of the psychophysical functions of sucrose, fructose, and the sucrose-fructose equiratio mixture are replotted with molarity as the measure of concentration, this appears not to be the case. We hold the view that comparisons should be made on the basis of molarity, not percentage of weight/volume, because molecules are the basic units for elicitation of a taste response.

\section{Conclusion}

Functional measurement in combination with the use of equiratio mixtures and a two-stimulus procedure provides a means of separating the sensory processes involved when mixing two taste substances from the judgmental process. In the present experiment, this methodology was used to derive psychophysical functions for glucose, fructose, and three equiratio mixture types of the two. The results confirm earlier findings that the taste intensity of a mixture of similar-tasting substances lies in between the intensities of the components when the concentration of the mixture and the concentrations of the components (outside the mixture) are the same.

\section{REFERENCES}

ANDERson, N. H. (1974). Algebraic models in perception. In E. C. Carterette \& M. P. Friedman (Eds.), Handbook of perception (Vol. 2 , pp. 215-298). New York: Academic Press.

ANDERSON. N. H. (1981). Foundations of information integration theor: New York: Academic Press.

ANDERSON, N. H. (1982). Methods of information integration theory. New York: Academic Press. 
Atrneave, F. (1962). Perception and related areas. In S. Koch (Ed.), Psychology: A study of science (Vol. 4, pp. 619-659). New York: McGraw-Hill.

BartoshuK, L. M. (1975). Taste mixtures: Is mixture suppression related to compression? Physiology \& Behavior, 14, 643-649.

BartoshuK, L. M. (1977). Psychophysical studies of taste mixtures. In J. Le Magnen \& P. MacLeod (Eds.), Olfaction and taste VI (pp. 377-384). London: I.R.L.

Birnbaum, M. H. (1980). Comparison of two theories of 'ratio' and 'difference' judgments. Journal of Experimental Psychology: General, 109, 304-319.

BIRNBAUM, M. H. (1982). Controversies in psychological measurement. In B. Wegener (Ed.), Social attitudes and psychophysical measurement (pp. 401-485). Hillsdale, NJ: Erlbaum.

Birnbaum, M. H., \& Elmasian, R. (1977). Loudness "ratios" and "differences" involve the same psychophysical operation. Perception \& Psychophysics, 22, 383-391.

Birnbaum, M. H., Mellers, B. A. (1978). Measurement and the mental map. Perception \& Psychophysics, 23, 403-408.

BirnbaUm, M. H., \& VeIT, C. T. (1974). Scale convergence as a criterion for rescaling: Information integration with difference, ratio, and averaging tasks. Perception \& Psychophysics, 15, 7-15.

BOGARTZ, R. S. (1980). Some functional measurement procedures for determining the psychophysical law. Perception \& Psychophysics, 27, 284-294.

Curtis, D. W., Attneave, F., \& Harrington, T. L. (1968). A test of a two-stage model of magnitude judgment. Perception \& Psychophysics, 3, 25-31.

Curtis, D. W., Stevens, D. A., \& Lawless, H. T. (1984). Perceived intensity of the taste of sugar mixtures and acid mixtures. Chemical Senses, 9, 107-120.

DE GraAF, C., \& FrIJTERs, J. E. R. (1986). A psychophysical investigation of Beidler's mixture equation. Chemical Senses, 11, 295-314.

DE GraAF, C., \& Fruters, J. E. R. (1987). Sweetness intensity of a binary sugar mixture lies in between intensities of its components, when each is tasted alone and at the same total molarity as the mixture. Chemical Senses, 12, 113-129.

Fristers, J. E. R. (in press). Psychophysical models for mixtures of tastants and mixtures of odourants. In S. D. Roper (Ed.), Olfaction and taste $I X$.

Fruters, J. E. R., De GraAf, C., \& Koolen, H. C. M. (1984). The validity of the equiratio taste mixture model investigated with sorbitolsucrose mixtures. Chemical Senses, 9, 241-248.

Fruters, J. E. R., \& OUde OPHUIS, P. A. M. (1983). The construction and prediction of psychophysical power functions for the sweetness of equiratio sugar mixtures. Perception, 12, 753-767.

Fruters, J. E. R., \& STEVENS, D. A. (1986). Psychophysical taste functions of equiratio acid mixtures. Manuscript submitted for publication.

Hagerty, M., \& Birnbaum, M. H. (1978). Nonmetric tests of ratios vs. subtractive theories of stimulus comparison. Perception \& Psychophysics, 24, 121-129.

KLITZNER, M. D. (1975). Hedonic integration: Test of a linear model. Perception \& Psychophysics, 18, 49-54.

MCBRIDE, R. L. (1982). Toward a unified theory of psychophysics. Doctoral dissertation, Macquari University, Sydney.

MCBride, R. L. (1986). The sweetness of binary mixtures of sucrose, fructose, and glucose. Journal of Experimental Psychology: Human Perception \& Performance, 12, 584-592.

McKenNa, F. P. (1985). Another look at the 'new psychophysics'. British Journal of Psychology, 76, 97-109.

Mellers, B. A., Davis, D. M., Birnbaum, M. H. (1984). Weight of evidence supports one operation for 'ratioss' and 'differences' of heaviness. Joumal of Experimental Psychology: Human Perception \& Performance, 10, 216-230.

Moskowitz, H. R. (1971). Intensity scales for pure tastes and for taste mixtures. Perception \& Psychophysics, 9, 51-56.

Moskowitz, H. R. (1973). Models of sweetness additivity. Journal of Experimental Psychology, 99, 88-98.

Moskowitz, H. R. (1974a). Models of additivity for sugar sweetness. In H. R. Moskowitz, B. Scharf, \& J. C. Stevens (Eds.), Sensation and measurement: Papers in honor of S. S. Stevens (pp. 379-388). Dordrecht, The Netherlands: Reidel.

MoskowItz, H. R. (1974b). Sourness of acid mixtures. Journal of Experimental Psychology, 102, 640-647.

RIFkIN, B., \& Bartoshuk, L. M. (1980). Taste synergism between monosodium glutamate and disodium 5'-guanylate. Physiology \& Behavior, 24, 1169-1172.

Rule, S. J., \& CuRTIS, D. W. (1977). Subject differences in input and output transformations from magnitude estimations of differences. Acta Psychologica, 41, 61-65.

Rule, S. J., CuRTIS, D. W., MARKLeY, R. P. (1970). Input and output transformations from magnitude estimation. Journal of Experimental Psychology, 86, 343-349.

ShePARD, R. N. (1981). Psychological relations and psychophysical scales: On the starus of 'direct' psychophysical measurement. Journal of Mathematical Psychology, 24, 21-57.

STEVENS, S. S. (1956). The direct estimation of sensory magnitudesloudness. American Journal of Psychology, 69, 1-25.

StONE, H., \& OLiver, S. M. (1969). Measurement of the relative sweetness of selected sweeteners and sweetener mixtures. Journal of Food Science, 34, 215-222.

Stone, H., Oliver, S. M., \& Kloehn, J. (1969). Temperature and ph effects on the relative sweetness of suprathreshold mixtures of dextrose fructose. Perception \& Psychophysics, 5, 257-260.

Torgerson, W. S. (1961). Distances and ratios in psychophysical scaling. Acto Psychologica, 19, 201-205.

Treisman, M. (1964). Sensory scaling and the psychophysical law. Quarterly Journal of Experimental Psychology, 16, 11-22.

van der Heyden, A., Brussel, L. B. P., Heidema, J. G., Kosmeyer, J. G., \& PEER, H. G. (1983). Interrelationships among synergism, potentiation, enhancement and expanded perceived intensity vs. concentration. Journal of Food Science, 48, 1192-1196.

VEIT, C. T. (1978). Ratio and subtractive processes in psychophysical judgment. Joumal of Experimental Psychology: General, 107, 81-107.

Weiss, D. J. (1972). Averaging: An empirical validity criterion for magnitude estimation. Perception \& Psychophysics, 12, 385-388.

Yamaguchi, S., Yoshikawa, T., IKeda, S., \& NinOmiYa, Y. (1970). Studies on the taste of some sweet substances: II. Interrelations among them. Agricultural \& Biological Chemistry, 34, 187-197.

(Manuscript received May 5, 1986; revision accepted for publication January $7,1987$. ) 